\title{
La cardiología, los cardiólogos y la recertificación
}

\author{
Dr. Enrique Soto
}

\author{
Palabras clave: $\quad$ CARDIOLOGÍA \\ CARDIÓLOGOS \\ RECERTIFICACIÓN
}

\section{Introducción}

La planificación de la atención sanitaria de un país está compuesta por un conjunto de variables, dentro de las cuales se encuentran los recursos humanos. Por otro lado, las enfermedades cardiovasculares registran una elevada prevalencia en la población, por lo cual es de interés analizar los aspectos relacionados al número de profesionales dedicados a la cardiología, su distribución geográfica, nivel de formación, disponibilidad y acceso a la actualización, y grado de satisfacción con respecto a las expectativas previstas en el desempeño de su profesión.

Los sistemas y las políticas de salud tienen por objeto brindar una adecuada asistencia sanitaria a la población de acuerdo a lo establecido por la evidencia científica y las posibilidades de cada país. Por esta razón es necesario incorporar al análisis otros factores tales como la conformación demográfica, la expectativa de vida, la prevalencia de los factores de riesgo cardiovascular y los aspectos sociales y económicos.

Los cambios demográficos, en particular el incremento de la expectativa de vida, tienen consecuencias en la epidemiología que, a su vez, hace necesario el análisis de los recursos humanos y materiales disponibles para las patologías que se estiman prevalentes.

Estos aspectos han sido motivo de diferentes foros de discusión como el de la Conferencia Europea sobre el Futuro de la Cardiología, la cual ha emitido la Declaración de Madrid en el año 2006(1).

Desde diversas publicaciones se ha prestado atención a esta temática, relacionada al número adecuado de profesionales en función de las demandas y al grado de formación y actualización de los mismos.

Recientemente se han desarrollado varias encuestas en Uruguay propiciadas por la Sociedad

\author{
Key words: \\ CARDIOLOGY \\ CARDIOLOGISTS \\ RECERTIFICATION
}

Uruguaya de Cardiología (SUC) y el Colegio Médico del Uruguay (CMU), cuyos resultados permiten analizar la realidad del país en cuanto a las variables cuantitativas y cualitativas relacionadas al profesionalismo médico en general y a la cardiología en particular.

\section{Para analizar la realidad}

$\mathrm{El}$ análisis de la realidad puede realizarse a partir de diferentes enfoques que se complementan ${ }^{(2)}$ :

a) Estimación de la oferta de médicos en general y de cardiólogos en particular. Para esto resulta imprescindible contar con una línea de base y una proyección que posibilite la realización de comparaciones con otros países de la región y el resto del mundo con la finalidad de aproximarnos al número adecuado de profesionales que se debe alcanzar para satisfacer las necesidades de la población y a sus niveles de formación y actualización.

b) Información sobre la demanda o utilización de los servicios de cardiología. En la actualidad, mediante la puesta en práctica de la historia clínica electrónica, resulta accesible establecer demoras, listas de espera en la consulta clínica con los especialistas, y posibilidades de acceso y retrasos en los estudios paraclínicos o inicio de tratamientos. En el futuro estos datos nos permitirán estimar con mayor precisión la situación de la atención cardiológica.

c) Análisis de las necesidades basadas en los cambios demográficos y en el diseño de las políticas de salud. Los cambios demográficos, la distribución geográfica y los acentos establecidos por las políticas de salud (por ejemplo en materia de 


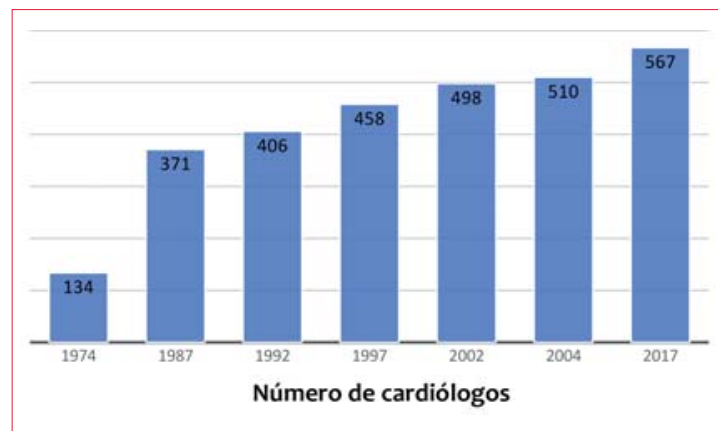

Figura 1. Evolución del número de cardiólogos.

prevención) contribuyen a establecer las necesidades en materia cuantitativa y cualitativa.

d) Recurrir a métodos basados en Benchmarking(3), en donde se buscan los referentes de buen funcionamiento a nivel internacional que se deseen imitar o sirvan como orientación para planificar acciones en beneficio de los objetivos que se desean alcanzar y de esta forma realizar las recomendaciones que se consideren necesarias.

\section{El análisis cuantitativo}

El CMU ha editado recientemente el libro La Profesión Médica en Uruguay(4), en donde se detallan aspectos cuantitativos. En el mismo, podemos observar la evolución del número de médicos, en donde se pasa de una densidad de 11,2 cada 10.000 habitantes en el año 1964 a 43,8 en el año 2017. Según los registros del CMU en Uruguay ejercen 15.298 médicos en la actualidad, que lo ubica dentro de los países con mayor número de profesionales de la región en relación con el número de habitantes.

En la mencionada publicación es posible observar algunas conclusiones consideradas de importancia. La distribución de los médicos en el territorio no es uniforme, mientras Montevideo cuenta con una densidad de 74,1 médicos cada 10.000 habitantes, el interior (globalmente) presenta 24,0. En cuanto a la estructura por edad y sexo se destaca que el $58,7 \%$ son mujeres y el $41,3 \%$ hombres. El proceso de feminización es más acentuado en las generaciones más jóvenes*.

Si bien no contamos con datos actualizados que nos permitan confirmar nuestra presunción, es muy probable que la conformación geográfica, etaria y de género tenga su correlato en la distribución de los cardiólogos en el país.

* Consulta personal a la Oficina de Registros de Títulos del Ministerio de Salud, en junio de 2018.
Tabla 1. Evolución del número de cardiólogos y su porcentaje en relación con el total de médicos.

\begin{tabular}{lcc}
\hline Año & $N$ & $\%$ \\
\hline 1974 & 134 & 3,4 \\
1987 & 371 & 4,6 \\
1992 & 406 & 4,2 \\
1997 & 458 & 4,1 \\
2002 & 498 & 3,8 \\
2004 & 510 & 3,9 \\
2017 & 567 & 3,3 \\
\hline
\end{tabular}

En los registros de títulos del Ministerio de Salud en la actualidad, figuran 16.815 habilitados para el ejercicio de la medicina en Uruguay, de los cuales 567 $(3,37 \%)$ se han especializado en cardiología. Según esta fuente de datos, en 30 años (1987-2017) se incrementó el número de cardiólogos en $34,5 \%$. No obstante, nuestro país carece de precisión en los registros de profesionales si se pretende una mayor rigurosidad en cuanto a las características y distribución de los médicos. Es de esperar que a partir de la base de datos que ha confeccionado el CMU mediante el carné único, sea posible albergar la información necesaria que permita aproximarnos a la realidad.

La evolución de los especialistas en cardiología en los últimos 44 años ha oscilado entre 3,4\% y 4,6\% del total de médicos habilitados para ejercer la profesión (figura 1, tabla 1).

De acuerdo al censo poblacional de Uruguay en 1975 había 2.788.429 habitantes, y en 2004 el registro ascendía a 3.241.603. En aproximadamente 30 años la población creció en $16,25 \%$, muy por debajo del incremento del número de cardiólogos.

La tasa de cardiólogos en la actualidad en Uruguay es de 17,49 cada 100.000 habitantes. La misma se encuentra dentro de las más altas en el contexto internacional, comparables a las de Grecia (el país con más elevado número de cardiólogos en relación con su población) ${ }^{(5)}$. Al tomar la República Argentina como ejemplo de la región, a los efectos de la comparación, observamos que en 2007 se encontraban registrados 7.468 cardiólogos, lo cual representa 195 cardiólogos por millón de habitantes ${ }^{(6)}$, levemente por encima de los datos correspondientes a nuestro país (174 por millón de habitantes) en la actualidad.

$\mathrm{Al}$ realizar una revisión bibliográfica nos encontramos con dificultades para establecer un número ideal de profesionales cardiólogos en relación con la población ${ }^{(7)}$, no obstante, si tenemos en cuenta el concierto internacional, Uruguay contaría con un número suficiente para satisfacer sus demandas. 
Evolución

- 1990: MSP. Proyecto de ley de recertificaciones médicas.

- 1990: Comisión de EMC del SMU.

- 1991: Consejo de la Facultad de Medicina aprueba proyecto de ley de "especialidades médicas".

- 1993: Encuentro de Solís de Entidades Médicas del Mercosur.

- 1993: Escuela de Graduados define la EMC dentro de sus fines.

- 1995: Se crea el Comité para la EMC.

- 1999: GET: MSP, Academia, Facultad de Medicina, SMU y FEMI. Acuerdo Marco.

- 2001: Comienza Acreditación de Instituciones y Actividades por Escuela de Graduados.

- 2004: Acuerdo Marco hacia una Ley de Educación Profesional.

- 2006: GETIP, Acuerdo Marco, se incorpora la Academia Nacional de Medicina.

- 2012: Colegio Médico del Uruguay. Se crea el CEPREM.

- 2012: La SUP aprueba la creación de una secretaría de recertificación.

- 2013: Encuesta Médica Nacional: 83\% de los médicos considera necesaria la recertificación.

EMC: Educación Médica Continua; SMU: Sindicato Médico del Uruguay; GET: Grupo de Trabajo Interinstitucional; FEMI Federación Médica del Interior; GETIP: Grupo de Trabajo Interinstitucional Permanente; CEPREM: Comisión de Educación Profesional y Recertificación Médica; SUP: Sociedad Uruguaya de Pediatría.

\section{La investigación cualitativa}

En el año 2009 la SUC le encargó a la socióloga Verónica Massonnier un estudio cualitativo que tenía por objeto investigar los niveles de satisfacción con respecto a las herramientas de comunicación: Revista Uruguaya de Cardiología, página web, correo SUC y actividades de formación. También se obtuvo información con respecto a las expectativas y la motivación acerca de la participación en la SUC(8).

La encuesta se realizó sobre una muestra representativa que comprendía a 50 cardiólogos entrevistados de manera telefónica sobre un cuestionario preestablecido, tanto de Montevideo como del interior del país.

Se deben destacar las interesantes conclusiones a las que arriba este estudio, entre las cuales se encuentra el valor que adquiere la revista de la SUC en la actualización y formación de los profesionales. También resultan de relevancia las diferentes per- cepciones sobre la necesidad de profundizar en la oferta formativa y en la búsqueda de alternativas para estimular la participación.

En el año 2017 la SUC resuelve realizar un nuevo análisis cualitativo con una muestra representativa de sus socios, con la intención de obtener información que permita avanzar en la idea de la recertificación de la especialidad. La misma se encargó nuevamente a la socióloga Massonnier, se entrevistaron 51 cardiólogos de acuerdo a una segmentación similar a la establecida en la encuesta del 2009 , con respecto a Montevideo e interior, mayores y menores de 45 años. El estudio abarcó tanto a quienes se desempeñaban en la cardiología clínica como en las subespecialidades ${ }^{(9)}$.

Los resultados de esta encuesta son de suma importancia y merecen un análisis detenido si deseamos una proyección de la especialidad de acuerdo a las necesidades de la población.

De la misma surgen tres etapas en la vida profesional del cardiólogo, cada una con características, valoraciones y necesidades diferentes: la etapa formativa, la de consolidación y la de madurez.

La etapa formativa, según el estudio, se extiende hasta los 40 años, en ella se produce un mayor contacto con la actividad académica, especial interés por la educación médica continua y la actualización de los conocimientos.

Le sigue la etapa denominada de consolidación, caracterizada por una intensa dedicación al ejercicio de la profesión; en este período no siempre es posible mantener el ritmo de actualización.

Por último, el estudio hace referencia a la etapa de madurez, en donde la conexión con el futuro es menor que en las anteriores, solo una minoría mantiene algún tipo de relación con la vida académica y surgen diferentes valoraciones del camino recorrido que van desde la satisfacción hasta el desencanto o la apatía.

Como también se había manifestado en la encuesta de 2009, aparecen factores relacionados a la distribución geográfica de los cardiólogos expresados como sensación de lejanía tanto real como simbólica.

También se hace referencia a un conjunto de situaciones que son percibidas como determinantes en el momento de establecer los grados de satisfacción: organización del trabajo (multiempleo), escaso tiempo para la vida familiar o social, falta de reconocimiento por parte de las instituciones. No obstante, se valora especialmente el reconocimiento en el trato directo de los pacientes.

Dentro de las conclusiones de la encuesta se hace referencia a los costos en términos de calidad de vida motivados por el modelo de trabajo. 


\section{La recertificación}

El profesionalismo médico comprende "el conjunto de conocimientos, habilidades, principios y valores que sustentan una práctica idónea de la medicina en el marco de los más elevados estándares de calidad científica, ética y humanística"(10).

La certificación constituye un requisito indispensable para el ejercicio de la profesión, ya que documenta que se han adquirido los conocimientos fundamentales, las habilidades y los valores para el ejercicio de la misma.

El dinamismo del desarrollo social y científico conduce a que los conocimientos adquiridos sufran transformaciones, y se produzcan cambios epidemiológicos, en las formas de diagnóstico y en los tratamientos de las enfermedades. La imposibilidad de acompañar estas transformaciones conduce a la obsolescencia $^{(11)}$.

La recertificación es el "resultado de un acto por el que una entidad médica legalmente acreditada y aplicando criterios preestablecidos asegura a través de un proceso de evaluación que un profesional médico previamente certificado mantiene actualizados sus conocimientos y destrezas, $\mathrm{y}$ ha desarrollado sus actitudes dentro del marco ético y científico adecuado de acuerdo con el progreso del saber y del hacer propio de su especialidad en un período determinado"(12).

En Uruguay, como en otros países, desde hace varios años, diferentes instituciones académicas y organizaciones científicas y gremiales han avanzado con la intención de progresar en el desarrollo profesional médico continuo con el objetivo de ofrecer una atención actualizada y de calidad a la población.

En la encuesta realizada por el CMU a los profesionales, el 81\% de los entrevistados consideraba como una de las funciones del mismo la implementación de la recertificación ${ }^{(13)}$.

La casi totalidad de los cardiólogos entrevistados en la Investigación Cualitativa de los Socios de la SUC, realizada en el año $2017^{(9)}$, afirmó que la recertificación le parecía importante o necesaria.

El dinamismo que ha adquirido la evolución de la investigación y los conocimientos en medicina, acompañados por el desarrollo de las nuevas tecnologías en comunicación, nos aproxima, cada vez con mayor necesidad, a resolver estos aspectos considerados esenciales para ofrecer una medicina de calidad.

\section{Bibliografía}

1. Escaned J, Ryden L, Zamorano J, Poole-Wilson P, Fuster V, Gitt A, et al. Tendencias y contextos en la práctica de la cardiología en los próximos 15 años. La Declaración de Madrid: un documento de la Conferencia Europea sobre el Futuro de la Cardiología, Madrid, 2-3 de junio de 2006. Rev Esp Cardiol. 2007;60(3):294-8.

2. Markham B, Birch S. Back to the future: a framework for estimating health-care human resource requirements. Can J Nurs Adm. 1997;10(1):7-23.

3. Costa JM. Benchmarking. Cuadernos de Gestión. $2000 ; 6: 68-7$.

4. Batthyany Dighiero K, Gonzalez F, Barbero M, Barrero G. La profesión médica en Uruguay. Montevideo: Colegio Médico del Uruguay; 2018.

5. Block P, Petch MC, Letouzey JP. Manpower in cardiology in Europe. Eur Heart J. 2000; 21(14): 1135-40 doi:10.1053/euhj.1999.1935

6. Borracci RA, Giorgi MA, Skon F, Hauad S, Guardiani FM, Lowenstein DM, et al. Estimación de la oferta y demanda de cardiólogos en la Argentina. Rev Arg Cardiol. 2009 [consulta: 17 Set 2018]; 77(1): [aprox.6p.]. Disponible en: https://www. sac.org.ar/wp-content/uploads/2014/04/1557.pdf

7. De Teresa Galván E, Alonso-Pulpón L, Barber P, Bover Freire R, Castro Beiras A, Cruz Fernández JM, et al. Desequilibrio entre la oferta y las necesidades de cardiólogos en España. Análisis de la situación actual, previsiones futuras y propuestas de solución. Rev Esp Cardiol. 2006 [consulta: 17 Set 2018];59(7): [aprox. 16p.]. Disponible en: http://www.revespcardiol.org/es/vol-59-num-07/sumario/13003944/

8. Massonier V. Sociedad Uruguaya de Cardiología. Investigación cualitativa de socios [diapositiva]. Montevideo: SUC;2009. 53 diapositivas.

9. Massonier V. Sociedad Uruguaya de Cardiología. Investigación cualitativa de socios [diapositiva]. Montevideo: SUC;2017. 42 diapositivas.

10. Wojtczak A. Profesionalismo Médico: Una problemática globlal. Educ Méd. 2006 [consulta: 17 Set 2018];9(3): [aprox.2p.]. Disponible en: http://scielo.isciii.es/pdf/edu/v9n3/original8.pdf

11. Dubin SS. Obsolescence or lifelong education: a Choice for the professional. American Psychologist 1972; 27(5):486-98. doi.org/10.1037/h0033050

12. Torres Calvete J. Certificación-recertificación profesional médica. Rev Méd Urug 2014 [consulta: 18 Set 2018]; 30(1): [aprox. 3p.]. Disponible en: http:// www.rmu.org.uy/revista/30/1/2/es/1/

13. Encuestas. 2016 La profesión médica en el Uruguay de hoy: percepciones, vivencias y expectativas. Montevideo: Colegio Médico del Uruguay; 2016 [consulta: 18 set 2018]. Disponible en: http://www.colegiomedico.org.uy/wp-content/uploads/2018/06/Encuestas.pdf 